\title{
Knowledge Sharing in a Large Agile Organisation: A Survey Study
}

\author{
Kati Kuusinen ${ }^{1(\bowtie)}$, Peggy Gregory ${ }^{1}$, Helen Sharp ${ }^{2}$, Leonor Barroca ${ }^{2}$, \\ Katie Taylor ${ }^{1}$, and Laurence Wood $^{3}$ \\ 1 University of Central Lancashire, Preston, UK \\ \{KKuusinen, AJGregory, KJTaylor\}@uclan.ac.uk \\ 2 The Open University, Walton Hall, Milton Keynes, UK \\ $\{$ Helen.Sharp, Leonor. Barroca\}@open.ac.uk \\ 3 IndigoBlue, London, UK \\ laurence.wood@indigoblue.co.uk
}

\begin{abstract}
Knowledge is a core resource for agile organisations that is transformed into products and services during the development process. Sharing of knowledge is essential across any organisation, and it has been claimed that the software industry requires more knowledge management than any other sector. Agile methodologies concentrate on team level collaboration, and some techniques for inter-team knowledge sharing have also proved to be successful. But these techniques focus on within-team and between-team knowledge sharing rather than knowledge sharing across the organisation. This paper presents the results of a survey with 81 responses on organisational knowledge sharing in a multinational agile company. The survey focuses on three aspects of knowledge sharing: within agile teams, beyond the team with company colleagues, and with customers. It concentrates on knowledge sharing practices, ease of knowledge sharing and motivation for knowledge sharing. Summary statistics, regression, and test of equity are used as analysis techniques. Results show that knowledge sharing with team members is significantly easier than with customers or company colleagues beyond their team. In addition, using agile practices improves ease of knowledge sharing within teams but not with customers or colleagues. Extrinsic motivators need to be in place to encourage knowledge sharing across the organisation, especially where such knowledge sharing is not an automatic consequence of completing the work.
\end{abstract}

Keywords: Knowledge sharing $\cdot$ Agile software development $\cdot$ Organisational knowledge sharing $\cdot$ Learning organisation

\section{Introduction}

Knowledge is awareness or understanding of something such as information or skills [4]. Knowledge creates most of the value in today's economy and the value of knowledge often increases when shared [23]. Organisational knowledge sharing

(c) The Author(s) 2017

H. Baumeister et al. (Eds.): XP 2017, LNBIP 283, pp. 135-150, 2017.

DOI: $10.1007 / 978-3-319-57633-6 \_9$ 
aims at transferring to the organisation the information, skills and experience a person or team has [10]. This is essential for sustaining the development of quality in software intensive companies [10]. For agile development companies, knowledge is the core resource that is transformed to products and services in the development process [2]. Moreover, Biao-wen [2] claims that the software industry requires more knowledge management than any other sector.

Agile methods focus heavily on the delivery of product and customer value. Moreover, an agile team focuses on applying knowledge instead of sharing it [10]. Agile methods facilitate knowledge sharing in the team but offer limited support for knowledge sharing outside the team $[6,17,18]$. Agile methods favour tacit knowledge shared informally using face-to-face communication (personalisation strategy) in contrast to traditional knowledge management practices [9]. Although attention has been paid to inter-team knowledge sharing [27], and techniques for distributed agile teams have proved to be successful, the focus here is on knowledge sharing across the organisation and not just between teams. The lack of knowledge sharing practices beyond the team can hinder sharing and sustaining knowledge in agile organisations [17].

This paper presents results of a baseline survey organised in a multinational agile software intensive company as part of their effort to improve organisational knowledge sharing. The results show that knowledge sharing with team members is significantly easier than with company colleagues or with customers. In addition, using more agile techniques is associated with increased ease of knowledge sharing with team members but not with colleagues outside the team and not with customers.

The rest of the paper is structured as follows: Sect. 2 introduces related research, Sect. 3 describes the research method, Sect. 4 presents the results, Sect. 5 considers limitations, Sect. 6 discusses the findings and Sect. 7 presents some conclusions and future work.

\section{Related Work}

Software engineering is a knowledge-intensive activity [25]. Software development teams are made up of knowledgeable individuals who need to be able to use, share, and communicate their knowledge in ways that foster problem solving and creativity. Whereas traditional software project approaches rely heavily on documentation and role-based working as ways of capturing and managing knowledge, agile approaches focus more on informal communication mechanisms within cross-functional teams $[6,10]$.

Agile approaches employ intensive team work, face-to-face knowledge sharing, and trust as vital elements of working practice [1]. Research evidence shows that good team work is crucial for project success, with important facets including communication, coordination, balance of member contributions, mutual support, effort and cohesion [15]. Studies of agile teams have found that agile practices improve both informal and formal communication, and facilitate team and organisational communication [22]. Information visibility and sharing 
are characteristics of agile approaches, especially when documentation is used. Sharp and Robinson [29] discuss how story cards and the Wall play an important part in the collaboration, co-ordination and communication processes of agile teams. Collaborative online tools are used to keep track of decisions and facilitate communication within collocated and distributed teams [8].

Knowledge management and learning theories have been used to explain the distinctiveness of the agile approach. Nonaka and Takeuchi's [21] distinction between explicit and tacit knowledge has been used to characterise the difference between traditional and agile approaches [6]. Explicit knowledge is objective, rational, and is easier to externalise in documents. In contrast, tacit knowledge is subjective, experience-based, and more likely to be context-specific and therefore easier to discuss than to document. Similarly, Hanssen et al. [14] identify two strategies for knowledge management: codification and personalisation. The codification strategy systematises and stores organisational knowledge, whereas the personalisation strategy supports the flow of information through the organisation through fostering connections between people and supporting a culture of communication. Traditional approaches tend towards codification whereas agile approaches tend towards personalisation.

Agile knowledge sharing practices can be roughly divided into practices among peers (e.g. communities of practice, pairing, coding dojos), among different specialists (shared specialists, interdisciplinary pairing, marathons), and among stakeholders and managers (scrum of scrums, review meetings). As agile becomes more widely adopted within companies and across industry, approaches for facilitating inter-team knowledge sharing and cross-organisational knowledge sharing need to be considered [3]. Inter-team personalisation strategies include Scrum of Scrums, project member rotation, communities of practice and open fishbowl sessions [27]. When viewed at an organisational level, knowledge is a significant competitive asset for a company. However, it is also challenging because of the scale and complexity of organisational environments and because the inter-team strategies do not address the needs of knowledge sharing across an organisation beyond teams collaborating in the same project.

Several authors identify that agile methods supply less advice for how to do this [6,17]. Santos et al. [27] propose a model showing how knowledge sharing between agile teams requires three elements: the adoption of practices, organisational support and appropriate stimuli. Recommended practices include face-to-face conversations, an informative workspace, rotation among teams and projects, collective meetings, pair programming between teams and projects, technical presentations, marathons, and coding dojos. Organisational support includes strategy, structure, culture, environment, top management and leadership support, communication flow and channels, integration among teams and projects, and deeper agile adoption. Appropriate stimuli include problems, common goals, incentives and sustainable pace. 


\section{Method}

The research goal for the study was to identify areas that require improvement in organisational knowledge sharing in an agile company and to provide a baseline for assessing the progress and effectiveness of future actions. The study was initiated by the company who approached the authors ${ }^{1}$ with a request to investigate their challenge. A survey ${ }^{2}$ was used to reach a wide audience, it was sent to company employees (not customers), and concentrated on knowledge sharing between three groups: team members, company colleagues, and customers. The research questions are as follows.

RQ 1 How is knowledge shared in the organisation?

RQ 2 What motivates knowledge sharing in the organisation?

RQ 3 Is there a relation between agility and ease of knowledge sharing?

RQ 4 Is there a relation between frequency of knowledge sharing activities and ease of knowledge sharing?

\subsection{Collaborator Company}

The company in which the survey was conducted is a large IT service provider that primarily develops software for UK customers but has staff distributed over three continents. The majority of their workforce is based in India, and are sent to work in development teams at customer sites on a temporary basis in several countries worldwide. Development teams are assigned to a specific customer account and thus have a strong customer focus in their job and dayto-day responsibilities; many teams are embedded in the customer organisation and hence distant from each other. While some cross-organisational knowledge sharing tools and practices have been put in place such as wikis, Yammer, and profession-specific groups for training, these are limited.

\subsection{Procedure}

The survey was developed iteratively in collaboration with our company contacts and piloted first with students and then with a few company representatives. A link to the online survey was then distributed via a contact person in the company and it was advertised on the company intranet. The survey was open from May to July 2016 and there were altogether 113 responses from company employees of which 81 were completed. Of the 81 complete responses, 36 responded to the open-ended question on how to improve knowledge sharing in the company. No incentives were offered and two reminders were sent. The survey was anonymous. Mean completion time was $11 \mathrm{~min}$ (SD $19 \mathrm{~min}$ ).

\footnotetext{
${ }^{1}$ The authors are members of the Agile Research Network (agileresearchnetwork.org) which is funded by the Agile Business Consortium Ltd. (ABC) Board, The Open University and University of Central Lancashire. Our research approach is explained here: Barroca, L., Sharp, H., Salah, D., Taylor, K., \& Gregory, P. (2015). Bridging the gap between research and agile practice: an evolutionary model. IJSA, 1-12.

2 The survey can be found from here: http://agileresearchnetwork.org/kss.
} 


\subsection{Survey}

The survey addressed practices, motivators and ease of knowledge sharing with team members, company colleagues and with the customer. The survey had three sections, on (1) agile methods and agile techniques employed, (2) knowledge sharing and (3) background information. Questions on knowledge sharing were related to frequency of use of knowledge sharing practices, motivation towards sharing and experienced ease of sharing. Survey themes were as follows

1. Agile methods employed (question 1, multiple choice)

2. Agile techniques employed (question 2, multiple choice)

3. Frequency of use of knowledge sharing practices with team members (question 3 , pre-defined list of practices assessed on four-point frequency scale)

4. Frequency of use of knowledge sharing practices with company colleagues outside the team (question 4, pre-defined list of practices assessed on fourpoint frequency scale)

5. Frequency of use of knowledge sharing practices with customer (question 5, pre-defined list of practices assessed on four-point frequency scale)

6. Motivation for knowledge sharing with team members, company colleagues and customer (question 6, multiple choice)

7. Ease of knowledge sharing with team members, with company colleagues outside the team and with customer (question 7, five-point Likert scale)

8. Suggestions for how to improve knowledge sharing in the company (question 8 , open-ended)

In addition we asked for background information including job role, years of experience in the company, the number of customer accounts and the number of people led if any.

The survey was designed to address the needs of the collaborator company and drew on existing literature. The first two questions on agile methods and techniques were adopted from the annual state of agile survey by Version One [31]. Question six on motivation was adapted from [19] and consisted of six statements measuring intrinsic and extrinsic motivation.

\subsection{Analysis}

We used basic descriptive statistics such as means to summarise responses on the structured questions. Since the data complied with the assumptions [5] of linear regression (F), a commonly used predictive analysis, we used it to study the relation between experienced ease of knowledge sharing and agility or frequency of knowledge sharing activities. We assumed that agility increases with the number of agile techniques employed. Gandomani et al. [11] propose a model and formula for calculating agility based on practices used. They use a list of 44 practices, of which ours is a sub-set. Thus, we use linear regression analysis to test whether experienced ease of knowledge sharing can be predicted from 
1. number of specific agile techniques employed (RQ 3)

2. reported frequency of use of knowledge sharing practices (RQ 4)

Based on Shapiro-Wilk test, the data was non-normal and thus we used a nonparametric hypothesis test. The selected Wilcoxon's signed rank test (Z) is a non-parametric statistical hypothesis test for comparing two related samples, e.g. two responses given by one single individual in a survey. We used the Wilcoxon test for equity to measure if there is a statistically significant difference between the experienced ease of knowledge sharing with team members, company colleagues and customers (RQ3, RQ4) and if there is a difference in the frequency of reporting motivation sources for sharing between those three groups (RQ2).

When sharing with either element of each of the partner pairs (team members and company colleagues, team members and customer, company colleagues and customer) the hypotheses are as follows:

1. there is no difference between the ease of knowledge sharing;

2. there is no difference between the frequency of intrinsic motivation sources;

3. there is no difference between the frequency of extrinsic motivation sources.

The hypotheses are a combination of the interests of the studied company and literature. For the open-ended question the data was collated and thematically analysed using an inductive, qualitative, data-driven content analysis with the aim of generating thematic groupings from the data [26], with no preconceived ideas about what would emerge.

\subsection{Respondents}

The response rate was $9 \%$. The main job responsibility of the 81 respondents was as follows: software development $42 \%$, architecture $16 \%$, project management $15 \%$, software testing or quality $7 \%$, business or system analyst $6 \%$, design or UX design $4 \%$, configuration/support $1 \%$ and other roles $9 \%$ (coaching or training or a mixture of development and design roles). Of the 81 respondents, $43 \%$ did not lead a team or function, 35\% led 1 to 9 persons, 14\% led 10 to 19 persons and $9 \%$ led over 19 persons. Almost all the respondents worked for customer accounts: $4 \%$ had not worked for a customer account, $30 \%$ had worked for one customer account, $40 \%$ for 2 to 4 customer accounts and $27 \%$ had worked for five or more customer accounts. On average, respondents had worked for the company for 7 years, standard deviation 6 years.

\section{Results}

For answers about agile methods and techniques multiple responses were possible. Scrum was the most used agile method reported by $83 \%$ of respondents. Kanban and Scrumban were also often used, reported respectively by $32 \%$ and $22 \%$ of the respondents. The most often employed agile techniques were daily standups, prioritised backlogs, iteration or sprint planning, retrospectives and short iterations or sprints (Fig. 1). 


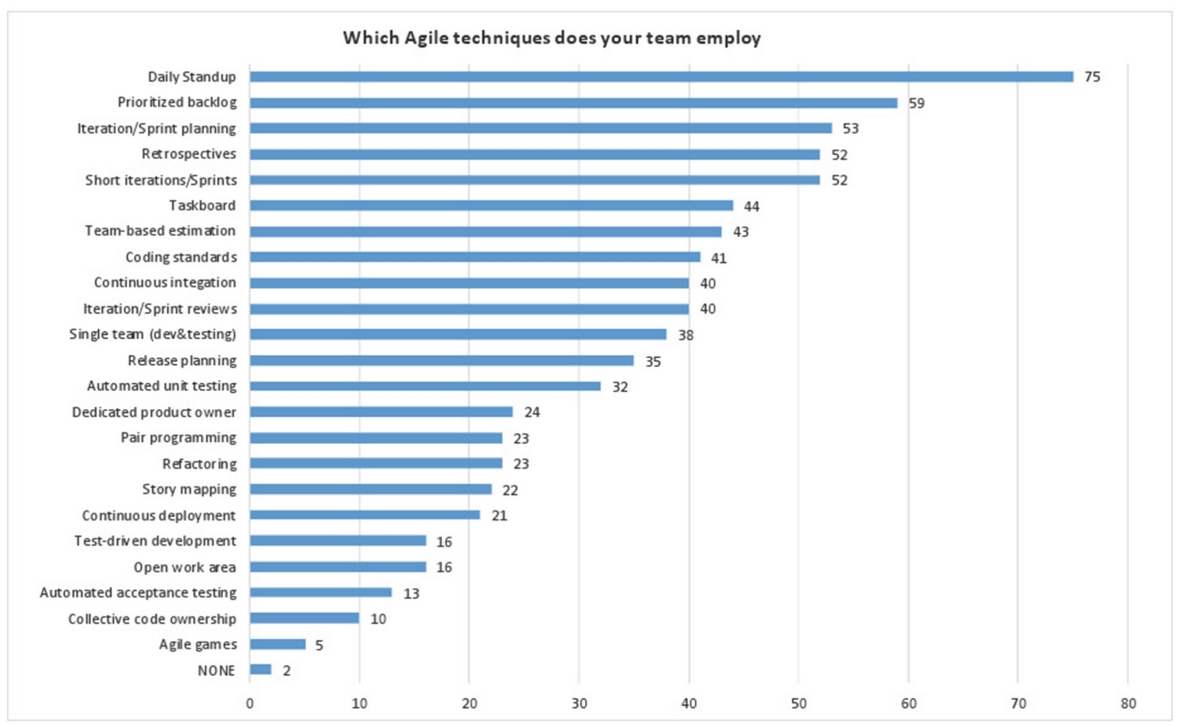

Fig. 1. Employed Agile techniques [31]

\subsection{Knowledge Sharing Practices}

The most common techniques for knowledge sharing in general were informally, in meetings, and by email (Fig. 2). In general, knowledge sharing was more frequent within teams than with customers or company colleagues outside the team. This is an expected result as teams are often the fundamental social units of an organisation's knowledge creation [16] and Scrum - the most widely used agile method in the company - emphasises the role of collaborative teams. Sharing knowledge with colleagues was most often done informally, whereas when sharing knowledge with customers, meetings were the most frequent technique. Both represent a personalisation knowledge sharing strategy (person-to-person) which is the favoured strategy in agile. The next most commonly used knowledge sharing techniques with customers were email and through the team lead or a senior member of the team.

\subsection{Motivation for Knowledge Sharing}

The mean number of reported motivation sources per respondent was higher for sharing knowledge with team members than with either company colleagues or customers (Fig. 3). There was a difference between the frequency of intrinsic and extrinsic motivators when sharing with customers compared to when sharing with either team members or company colleagues. When sharing knowledge with team members or company colleagues, a greater number of respondents reported intrinsic sources of motivation than extrinsic sources whereas when sharing with 


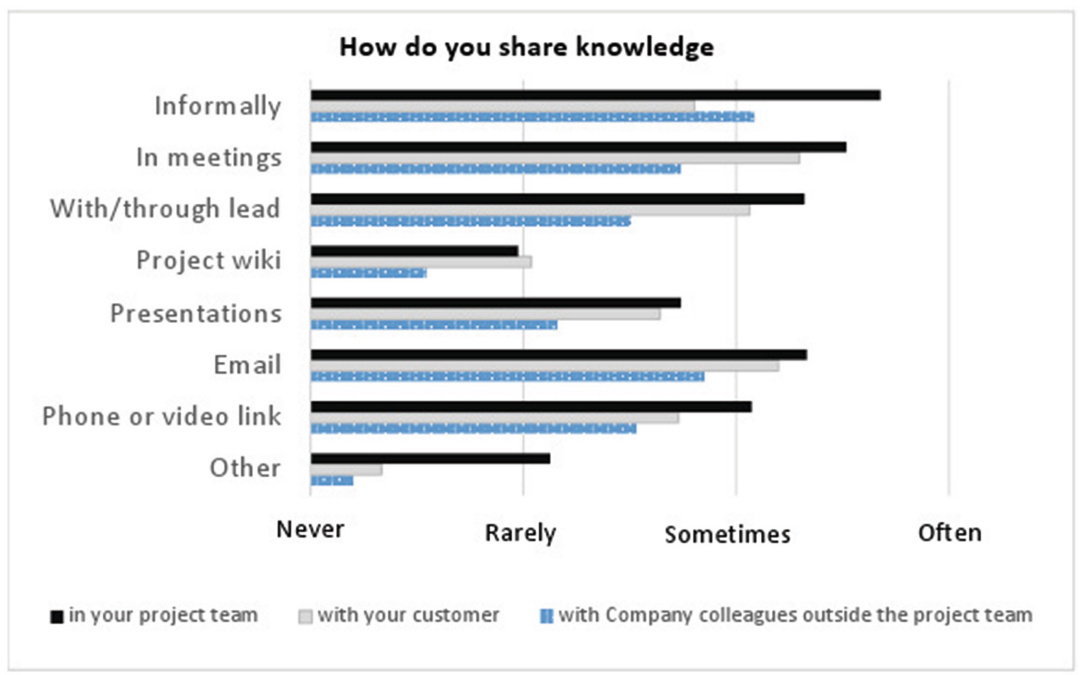

Fig. 2. Mean frequency of use of knowledge sharing practices in team, in company and with customer. $\mathrm{N}=81$

Table 1. Percentage of respondents reporting motivation source types per sharing partner. $\mathrm{N}=81$.

\begin{tabular}{l|r|l|l}
\hline Motivation source & Team & Colleague & Customer \\
\hline Both extrinsic and intrinsic & $85 \%$ & $63 \%$ & $59 \%$ \\
\hline Intrinsic only & $14 \%$ & $16 \%$ & $10 \%$ \\
\hline Extrinsic only & $1 \%$ & $10 \%$ & $21 \%$ \\
\hline None & $0 \%$ & $11 \%$ & $10 \%$ \\
\hline
\end{tabular}

customers a greater number of respondents reported extrinsic sources of motivation than intrinsic sources (Table 1 ).

Enjoyment was the most common motivator for knowledge sharing with team members (90\% of respondents mentioned it) and with company colleagues $(67 \%)$ whereas with customer it was strengthening ties (64\%) (Fig. 3). Enjoyment is an intrinsic motivator whereas strengthening ties is an extrinsic motivator [16,19].

The Wilcoxon signed rank test was applied to the data. Based on the results, all hypotheses considering motivation sources were rejected apart from the following: there is no difference between the frequency of intrinsic motivation sources (1) when sharing with company colleagues and (2) when sharing with customers (Table 2). However there is a significant difference in the frequency of reporting extrinsic motivation sources between sharing knowledge with company colleagues and customers. The most obvious difference is that strengthening ties was an especially frequent source of motivation for sharing knowledge with customers, which is important for maintaining the relationship with the customer. 
Table 2. Wilcoxon signed rank test on the frequency of motivation sources for sharing knowledge with team members, company colleagues and customer. $\mathrm{N}=81$.

\begin{tabular}{l|l|l}
\hline Compared sharing partner pair & Test outcome $(\mathrm{Z})$ & Level of significance $(\mathrm{p})$ \\
\hline Intrinsic: Team members - Colleagues & $\mathrm{Z}=-3.98$ & $\mathrm{p}<.001$ \\
\hline Intrinsic: Team members - Customer & $\mathrm{Z}=-4.94$ & $\mathrm{p}<.001$ \\
\hline Intrinsic: Colleagues - Customer & $\mathrm{Z}=-1.53$ & n.s. \\
\hline Extrinsic: Team members - Colleagues & $\mathrm{Z}=-4.12$ & $\mathrm{p}<.001$ \\
\hline Extrinsic: Team members - Customer & $\mathrm{Z}=-2.33$ & $\mathrm{p}<.05$ \\
\hline Extrinsic: Colleagues - Customer & $\mathrm{Z}=-2.00$ & $\mathrm{p}<.05$ \\
\hline
\end{tabular}

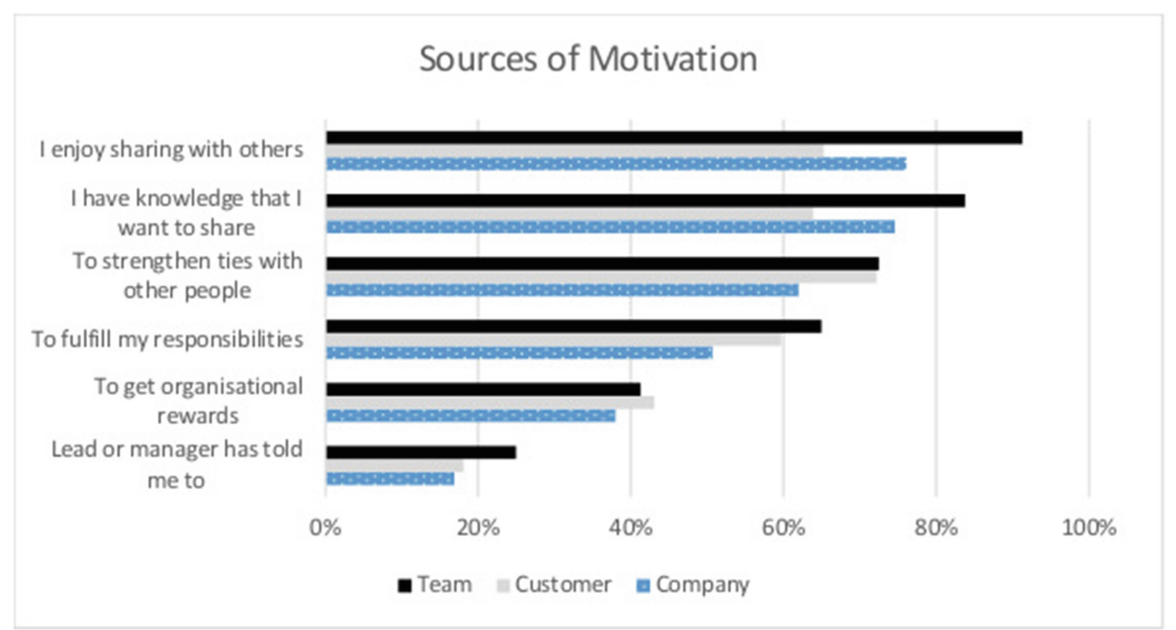

Fig. 3. Frequency of motivation sources for knowledge sharing with team members, customer and company colleagues outside the team. $\mathrm{N}=81$.

In summary, this test showed differences between the frequencies of extrinsic motivation sources for sharing with all the sharing partners and between the frequencies of intrinsic sources between all the sharing partners except company colleagues and the customer.

\subsection{Ease of Knowledge Sharing}

Knowledge sharing within teams was reported to be easy whereas knowledge sharing beyond the team with company colleagues and with customers was less easy (Fig. 4). A Wilcoxon signed rank test was applied to the findings. This revealed that knowledge sharing with team members was significantly easier than with customers $(Z=-4.51, p<.001)$. It also revealed that knowledge sharing with team members was significantly easier than with company colleagues outside the team $(Z=-4.52, p<.001)$. Based on the test, the hypotheses there 
is no difference between the ease of knowledge sharing with team members and customers and there is no difference between the ease of knowledge sharing with team members and company colleagues were rejected while the hypothesis there is no difference between the ease of knowledge sharing with company colleagues and customers was accepted. Of the respondents, $62 \%$ strongly agreed that knowledge sharing with team members is easy whereas $28 \%$ and $27 \%$ strongly agreed that knowledge sharing with customers or with company colleagues, respectively, is easy. Knowledge sharing with customers was considered slightly easier than with company colleagues (Fig. 4). Only $9 \%$ did not agree that knowledge sharing is easy with team members whereas $30 \%$ did not agree that knowledge sharing is easy with customers and $33 \%$ did not agree that knowledge sharing is easy with company colleagues outside the team. Thirty-six employees suggested improvements for organisational knowledge sharing in an open-ended question. Almost all of the suggestions were about knowledge sharing in the company outside the team. Half of the respondents suggested having small informal sessions among interested individuals to share knowledge, for example, about architectural solutions or new technologies. Also, half of respondents suggested either creating new knowledge bases, or repositories, or using the current ones more efficiently. Other ideas included fostering the company culture to embrace knowledge sharing. Such a culture would build on trust and encourage people to share their knowledge instead of making them fear they are replaceable if they share.

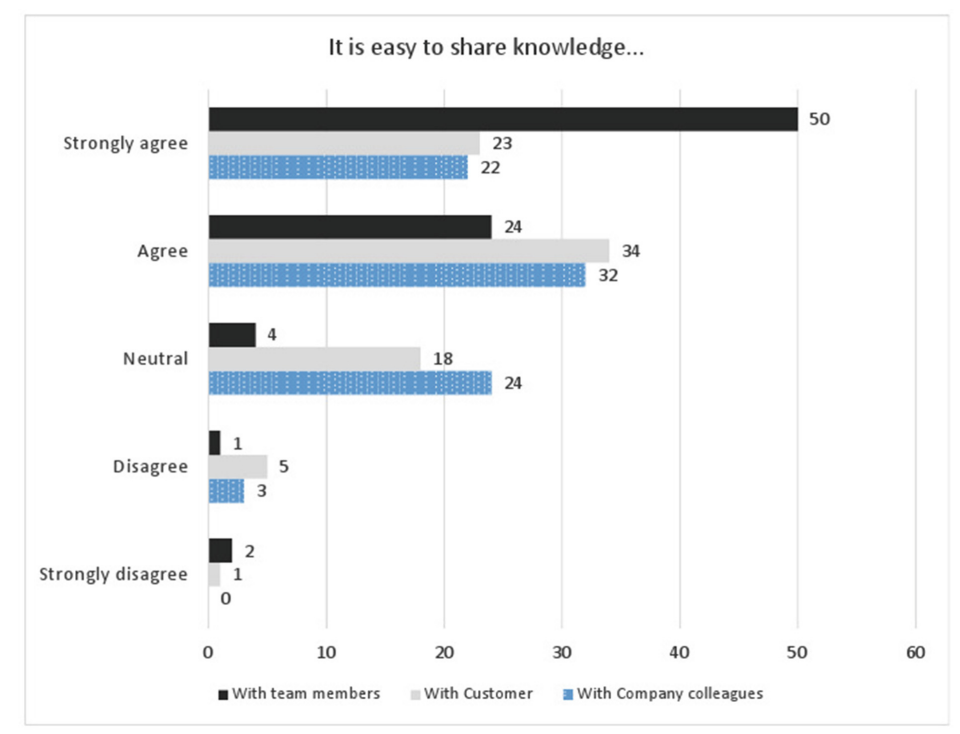

Fig. 4. Perceived ease of knowledge sharing with team members, customer and colleagues. $\mathrm{N}=81$. 


\subsection{Relation of Agility and Ease of Knowledge Sharing}

Experienced ease of knowledge sharing with team members could be predicted from the number of agile techniques employed using linear regression $F(80,1)=$ $10.7, p<.01$. Thus, the greater the number of agile techniques employed, the easier knowledge sharing with team members was experienced.

There is no direct association between the number of agile techniques employed and experienced ease of knowledge sharing with company colleagues, $F(80,1)=0.0, n . s$, nor between the number of agile techniques employed and experienced ease of knowledge sharing with customers, $F(80,1)=2.7$, n.s.

\subsection{Relation of Frequency and Ease of Knowledge Sharing}

There is a direct association between the frequency of use of knowledge sharing practices and experienced ease of knowledge sharing with team members: the more frequently knowledge sharing practices are used, the easier knowledge sharing is, $F(78,12)=3.6, p<.001$. When ease of knowledge sharing with team members was calculated from knowledge sharing practices, using the whiteboard $(t=3.8, p<.001)$ and doing it informally $(t=2.8, p<.01)$ are significant positive predictors whereas using Yammer $(t=-2.0, p<.05)$ was a significant negative predictor. Thus, the more frequently whiteboards are used for knowledge sharing or the more frequently knowledge is shared informally with team members, the easier knowledge sharing with team members is experienced. On the contrary, the more often knowledge is shared via Yammer with team members, the less easy knowledge sharing with team members is experienced.

Using a whiteboard requires face-to-face contact whereas Yammer moves people away from physical presence, which may explain the negative association. These results indicate that knowledge sharing is easier where frequent, informal sharing takes place, including using whiteboards as a knowledge sharing tool.

Experienced ease of knowledge sharing with company colleagues can be predicted from the frequency of use of knowledge sharing practices using multiple linear regression, $F(80,11)=1.9, p<.05$. When ease of knowledge sharing with colleagues was calculated from knowledge sharing practices, giving presentations $(\mathrm{t}=-2.0, \mathrm{p}<.05)$ was a significant negative predictor.

In general, the more frequently knowledge sharing practices are used, the easier knowledge sharing appears to be. However, giving presentations is a negative predictor. A possible explanation for this negative association is that presentations are often one-directional: the presenter shares their information with the audience. Furthermore, the company also shares presentations via email. Using one-directional practices for knowledge sharing may decrease the experienced ease of knowledge sharing.

There is a direct association between the frequency of use of knowledge sharing practices and the experienced ease of knowledge sharing with customers. Experienced ease of knowledge sharing with customers can be predicted from the frequency of use of knowledge sharing practices using multiple linear regression, $F(80,7)=5.8, p<.001$. When ease of knowledge sharing with customers 
was calculated from knowledge sharing practices, using a wiki $(t=3.6, p<.01)$ and having meetings $(t=2.6, p<.05)$ were significant predictors. Thus, knowledge sharing is easier when a collaborative exchange of information is frequently used and meetings with the customer are frequent.

\section{$5 \quad$ Limitations}

Construct validity relates to the appropriateness of the survey as a measure. Several techniques were used to mitigate this threat. Questions 1, 2 and 6 in the survey were based on questions found in existing literature, to ensure that terminology used was in common use. The survey was developed iteratively and piloted with practitioners. Some of the questions contained repetition asking respondents to consider knowledge sharing from three perspectives, with team members, company colleagues and customers. Factors such as boredom and practice could have impacted the results. Question randomisation or counterbalancing were not used because of limitations of the surveying tool. Multiple response was controlled by allowing only one response per device. Internal validity relates to causal conclusions drawn. We used the number of specific agile techniques employed as a measure of agility in the survey, an approach used by $[11,24]$. This is not sophisticated, however in the context of this survey it provides a useful indication of how agility varies within the company. The strength of motivators was not asked for and therefore it is unknown if some of them are more powerful than the others. The measures of agility and motivation were both used in the linear regression analysis. Moreover, statistical tests are always prone to incorrect rejection or retaining of the null hypothesis and multiple hypothesis testing increases the risk. We did not use adjustments for these error types since correction of one of the types increases the risk to the other type. External validity relates to generalizability of the findings. As only one company was surveyed, the results are specific to that company. Moreover, only a number of employees responded to the survey which makes it prone to non-response bias.

\section{Discussion}

The summary answers to our research questions are as follows:

RQ 1 How is knowledge shared in the organisation? The top three knowledge sharing practices are: sharing informally, in meetings, and through email. Sharing knowledge with colleagues is most often done informally whereas with customers the most common means is in meetings.

RQ 2 What motivates knowledge sharing in the organisation? Respondents cited more motivators for sharing with team members than with company colleagues or customers. Motivators for knowledge sharing with team members and with company colleagues are more frequently intrinsic than extrinsic; motivators for knowledge sharing with customers are more frequently extrinsic. 
RQ 3 Is there a relation between agility and ease of knowledge sharing? Sharing knowledge within teams is statistically significantly easier than with customers or company colleagues. The regression analysis shows that using agile techniques improves ease of knowledge sharing within agile teams but not with company colleagues or with customer.

RQ 4 Is there a relation between the frequency of knowledge sharing activities and ease of knowledge sharing? In general the more frequently knowledge sharing practices are used, the easier knowledge sharing is. However, there are nuances in the data with some practices improving knowledge sharing and some hindering it. Our findings suggest that knowledge sharing is easier if face-to-face and informal contact is used, whereas one-way presentations decrease the perceived ease of knowledge sharing.

Our findings indicate that specific agile techniques improve ease of knowledge sharing within teams. This confirms findings from Pikkarainen et al. [22] who found that agile practices improved both informal and formal communication, and [20], who suggest that the "knowledge-as-relationship" focus of agile teams facilitates team knowledge sharing. It also confirms common-sense expectations that agility improves knowledge sharing and communication within the team.

Our findings also suggest that a high level of agility helps knowledge sharing to some extent with customers, but has little impact on knowledge sharing with company colleagues. This finding confirms the view that simply using agile techniques does not help much with inter-team knowledge sharing $[6,17]$.

Software engineers are outcome-oriented and motivated by technically interesting content and the work itself [28]. One of the characteristics of agile working is that all of the team's effort is focused on producing code that provides business value, and that plays directly into this motivation profile. In this context, sharing experiences with company colleagues who are not directly involved in the same project or with the same customer, requires compelling extrinsic motivators. Yet motivators for knowledge sharing with company colleagues were intrinsic rather than extrinsic. Therefore, it seems clear that this organisation does not have sufficient extrinsic motivators in place to encourage knowledge sharing with company colleagues.

These results are influenced by the collaborator's specific cicumstances, and these require further investigation. For example they are mostly based in India and the Indian agile community faces a range of challenges [30], are embedded in customer sites around the world, and hence at a distance from each other.

\section{Conclusions and Future Work}

Our survey study contributes to an understanding of how knowledge is shared in agile organisations. We provide evidence to support claims that knowledge sharing is easier within agile teams. In this instance, we find that these benefits do not apply to knowledge sharing across the organisation. Extrinsic motivators need to be in place to encourage knowledge sharing across the organisation, 
especially where such knowledge sharing is not an automatic consequence of completing the work.

Further research is required to investigate how knowledge sharing may be improved across this organisation, to compare their situation with other companies, and to understand better how the organisation's specific situation influences knowledge sharing behaviour. Suggestions from literature will be used to guide the next stage, for example ecosystems, communities of practice, shared specialists, coding marathons and project members' rotation $[6,7,27,32]$. Santos et al's [27] model of inter-team knowledge sharing suggests that three elements are important in inter-team knowledge sharing: the adoption of specific practices, organisational support and appropriate stimuli. Some of their suggestions for practices, such as job rotation, role pairing between projects and informal crossorganisational networks are not currently in place, but could be introduced. Han and Anantatmula's [13] model for knowledge sharing in large IT organisations identifies organisation, technology, learning and leadership as important components. Their suggestions for leadership highlight the importance of aspects such as a management help with knowledge sharing, verbal praise, encouragement, and career promotion. These observations could be characterised as cultural issues, such as those identified in [12].

\section{References}

1. Beck, K., Beedle, M., van Bennekum, A., Cockburn, A., Cunningham, W., Fowler, M., et al.: The Agile Manifesto (2001)

2. Biao-wen, L.: The analysis of obstacles and solutions for software enterprises to implement knowledge management. In: 2010 The 2nd IEEE International Conference on Information Management and Engineering (ICIME), pp. 211-214. IEEE (2010)

3. Bjørnson, F.O., Dingsøyr, T.: Knowledge management in software engineering: a systematic review of studied concepts, findings and research methods used. Inf. Softw. Technol. 50(11), 1055-1068 (2008)

4. Charband, Y., Navimipour, N.J.: Online knowledge sharing mechanisms: a systematic review of the state of the art literature and recommendations for future research. Inf. Syst. Front., pp. 1-21 (2016)

5. Chatterjee, S., Simonoff, J.S.: Handbook of Regression Analysis, vol. 5. Wiley, New York (2013)

6. Chau, T., Maurer, F., Melnik, G.: Knowledge sharing: Agile methods vs. Tayloristic methods. In: WETICE, vol. 3, pp. 302-307 (2003)

7. Cockburn, A., Highsmith, J.: Agile software development, the people factor. Computer 34(11), 131-133 (2001)

8. Deshpande, A., Sharp, H., Barroca, L., Gregory, P.: Remote working and collaboration in agile teams. In: International Conference on Information Systems, ICIS 2016. AIS Electronical Library (2016)

9. Dybå, T., Dingsøyr, T.: Empirical studies of agile software development: a systematic review. Inf. Softw. Technol. 50(9), 833-859 (2008)

10. Ersoy, I.B., Mahdy, A.M.: Agile knowledge sharing. Int. J. Softw. Eng. (IJSE) 6(1), $1-15(2015)$ 
11. Gandomani, T.J., Nafchi, M.Z.: An empirically-developed framework for agile transition and adoption: a grounded theory approach. J. Syst. Softw. 107, 204-219 (2015)

12. Gregory, P., Barroca, L., Sharp, H., Deshpande, A., Taylor, K.: The challenges that challenge: engaging with agile practitioners concerns. Inf. Softw. Technol. 77, 92-104 (2016)

13. Han, B.M., Anantatmula, V.S.: Knowledge sharing in large it organizations: a case study. Vine 37(4), 421-439 (2007)

14. Hansen, M.T., Nohria, N., Tierney, T.: Whats your strategy for managing knowledge? In: The Knowledge Management Yearbook 2000-2001, pp. 55-69 (1999)

15. Hoegl, M., Gemuenden, H.G.: Teamwork quality and the success of innovative projects: a theoretical concept and empirical evidence. Organ. Sci. 12(4), 435-449 (2001)

16. Hung, S.Y., Durcikova, A., Lai, H.M., Lin, W.M.: The influence of intrinsic and extrinsic motivation on individuals' knowledge sharing behavior. Int. J. Hum. Comput. Stud. 69(6), 415-427 (2011)

17. Karlsen, T.J., Hagman, L., Pedersen, T.: Intra-project transfer of knowledge in information systems development firms. J. Syst. Inf. Technol. 13(1), 66-80 (2011)

18. Kettunen, P., Laanti, M.: Combining agile software projects and large-scale organizational agility. Softw. Process Improv. Pract. 13(2), 183-193 (2008)

19. Lin, H.F.: Effects of extrinsic and intrinsic motivation on employee knowledge sharing intentions. J. Inf. Sci. 33(3), 340-359 (2007)

20. Melnik, G., Maurer, F.: Direct verbal communication as a catalyst of agile knowledge sharing. In: Agile Development Conference 2004, pp. 21-31. IEEE (2004)

21. Nonaka, I., Takeuchi, H.: The Knowledge-Creating Company: How Japanese Companies Create the Dynamics of Innovation. Oxford University Press, New York (1995)

22. Pikkarainen, M., Haikara, J., Salo, O., Abrahamsson, P., Still, J.: The impact of agile practices on communication in software development. Empirical Softw. Eng. 13(3), 303-337 (2008)

23. Quinn, J.B., Anderson, P., Finkelstein, S.: Managing professional intellect: making the most of the best. In: Strategic Management of Intellectual Capital, pp. 87-100 (1998)

24. Qumer, A., Henderson-Sellers, B.: An evaluation of the degree of agility in six agile methods and its applicability for method engineering. Inf. Softw. Technol. 50(4), 280-295 (2008)

25. Qumer, A., Henderson-Sellers, B.: A framework to support the evaluation, adoption and improvement of agile methods in practice. J. Syst. Softw. 81(11), 1899-1919 (2008)

26. Ritchie, J., Lewis, J., Nicholls, C.M., Ormston, R., et al.: Qualitative Research Practice: A Guide for Social Science Students and Researchers. Sage (2013)

27. Santos, V., Goldman, A., De Souza, C.R.: Fostering effective inter-team knowledge sharing in agile software development. Empirical Softw. Eng. 20(4), 1006-1051 (2015)

28. Sharp, H., Baddoo, N., Beecham, S., Hall, T., Robinson, H.: Models of motivation in software engineering. IST 51(1), 219-233 (2009)

29. Sharp, H., Robinson, H.: Three 'C's of Agile Practice: Collaboration, Co-ordination and Communication. In: Dingsøyr, T., Dybå, T., Moe, N.B. (eds.) Agile Software Development, pp. 61-85. Springer, Heidelberg (2010) 
30. Srinivasan, J., Lundqvist, K.: Agile in India: challenges and lessons learned. In: Proceedings of ISEC 2010 the 3rd India Software Engineering Conference, pp. 125-130. ACM, New York (2010)

31. VersionOne: Annual State of Agile Survey (2016). http://stateofagile.versionone. com. Accessed 29 Nov 2016

32. Wenger, E., McDermott, R.A., Snyder, W.: Cultivating Communities of Practice: A Guide to Managing Knowledge. Harvard Business Press (2002)

Open Access This chapter is licensed under the terms of the Creative Commons Attribution 4.0 International License (http://creativecommons.org/licenses/by/4.0/), which permits use, sharing, adaptation, distribution and reproduction in any medium or format, as long as you give appropriate credit to the original author(s) and the source, provide a link to the Creative Commons license and indicate if changes were made.

The images or other third party material in this chapter are included in the chapter's Creative Commons license, unless indicated otherwise in a credit line to the material. If material is not included in the chapter's Creative Commons license and your intended use is not permitted by statutory regulation or exceeds the permitted use, you will need to obtain permission directly from the copyright holder.

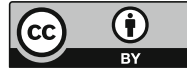

